\title{
Nickel exposure from keys: a Brazilian issue*
}

\author{
Nathalie Mie Suzuki ${ }^{1}$ \\ Mariana de Figueiredo Silva Hafner ${ }^{1}$
}

\author{
Ida Alzira Gomes Duarte ${ }^{1,2}$ \\ Rosana Lazzarini ${ }^{1,2}$
}

\section{DOI: http://dx.doi.org/10.1590/abd1806-4841.20175754}

\begin{abstract}
Keys are a significant source of exposure to metal allergens and can be a relevant problem for nickel-allergic individuals. This study aimed to perform nickel and cobalt spot testing among the 5 most common Brazilian brands of keys. Among the tested keys, $100 \%$ showed positive result to nickel spot test, $83,3 \%$ presented strong positive reaction. $50 \%$ exhibited cobalt release as well. Nickel release from keys is very common in our country and may cause a negative impact on sensitized individual's quality of life. Study's results highlight the importance of establishing directives to regulate nickel release in Brazil.
\end{abstract}

Keywords: Cobalt; Dermatitis, allergic contact; Metals; Nickel

In several published studies, nickel is the main sensitizer for children, adolescents and adults. Frequency of nickel allergy reaches approximately $20 \%$ in several communities, making it the main agent of contact allergic dermatitis (CAD). ${ }^{1}$ It is present in a great variety of objects of our daily life.

Another important agent of CAD is cobalt, also present in several metallic objects and associated with nickel in many of them. Association of positive contact tests with nickel and cobalt is common.

Thus, keys, present in the daily routine of everyone, can be a source of sensitization to nickel and cobalt for susceptible individuals. Repeated and constant exposure to different types of keys favors exacerbations or even persistence of CAD.

Release of nickel by metallic objects can be detected by dimethylglyoxime (DMG) or nickel spot test at levels starting at 10 ppm, a threshold already established as capable of inducing CAD. When the material shows nickel release above this level, the cotton soaked with dimethylglyoxime, rubbed into the metal, turns pink. ${ }^{2}$

Release of cobalt from objects is determined by a similar test (cobalt spot test), performed with Nitroso-R salt. Presence of cobalt at concentrations from $8.3 \mathrm{ppm}$ leads to a yellow-orange color. ${ }^{3}$

The objective of this study was to verify the presence of nickel and cobalt in different types of keys used by our population, through nickel and cobalt spot test, capable of identifying those with the capacity to induce or trigger CAD. Flexible cotton-tipped rod was used for testing. Four Brazilian key brands (Jaz, do Vale,
Stam and Gold) of the most used models (Yale and Tetra) were tested. Eight keys were tested in total.

The Yale model, which uses a four- to eight-pin cut, is used for residential door locks and padlocks, drawers and cabinets.

The Tetra model has four sides, each with a cut of four- to eight-pins. Because it is safer, it is used on residential and commercial doors.

All tested keys were new, obtained from a building materials store (Figure 1).

Each key was tested in its main areas, being rubbed for 30 seconds. The spot tests were conducted in different areas of the keys to avoid false positives.

The nickel test was considered strongly positive when cotton became solid pink; weakly positive, when it presented incomplete pink staining; and negative when no staining change was observed (Figure 2). The cobalt test had positive reaction when it presented orange staining, and negative when there was no color change.

Among the tested keys, $100 \%$ presented positive tests for nickel release, being $83.3 \%$ with strongly positive reaction. Regarding cobalt, $50 \%$ of the tested keys also showed positive cobalt test.

In Brazil, there is no regulation for the presence of metals in materials. This contributes to high levels of sensitization to nickel and cobalt. ${ }^{1,4,5}$ Brazilian frequency of CAD is approximately $28.16 \%$ for nickel and $10.52 \%$ for cobalt.

Received on 02.03.2016.

Approved by the Advisory Board and accepted for publication on 06.07.2016.

* Study conducted at the Dermatology Clinic of the Santa Casa de São Paulo - São Paulo (SP), Brazil.

Financial support: none.

Conflict of interest: none.

Dermatology Clinic of the Santa Casa de São Paulo - São Paulo (SP), Brazil.

School of Medical Sciences of the Santa Casa de São Paulo (FCMSCSP) - São Paulo (SP), Brazil.

(C)2017 by Anais Brasileiros de Dermatologia 


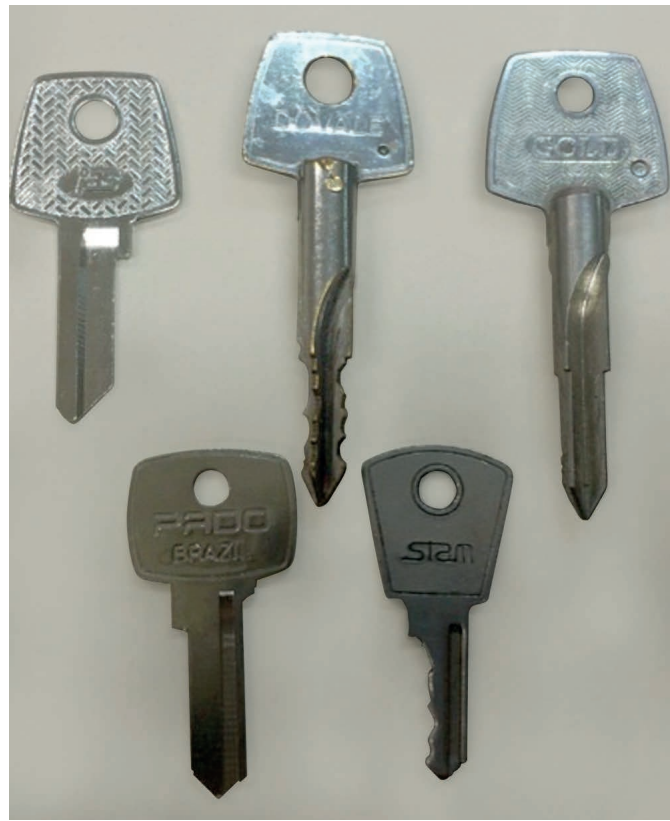

FIGURE 1: Tetra and Yale models of the leading brands of Brazilian keys tested

In nature, cobalt, nickel and copper coexist. Thus, in metallic alloys containing nickel, there is also cobalt, which results in concomitant exposure and sensitization.

Nickel sensitization remains the leading cause of CAD and, according to an American review, it has increased exponentially over the past five decades. ${ }^{6}$ This increase is justified by the change in consumer exposure. In the last decade, there was a popularization of the use of portable electronic equipment, which contributed to the exposure to devices with nickel release. This was evident mainly in the infant population, in contact with these equipments in an increasingly precocious way.

\section{REFERENCES}

1. Duarte IA, Tanaka GM, Suzuki NM, Lazzarini R, Lopes AS, Volpini BM, et al. Patch test standard series recommended by the Brazilian Contact Dermatitis Study Group during the 2006-2011 period. An Bras Dermatol. 2013;88:1015-8.

2. Hamann D, Scheman AJ, Jacob SE. Nickel exposure from keys: alternatives for protection and prevention. Dermatitis. 2013;24:186-9.

3. Thyssen JP. Cobalt sensitization and dermatitis: considerations for the clinician. Dermatitis. 2012;23:203-9.

4. Rodrigues DF, Goulart EM. Patch test results in children and adolescents. Study from the Santa Casa de Belo Horizonte Dermatology Clinic, Brazil, from 2003 to 2010.. An Bras Dermatol. 2015;90:671-83.

5. Rodrigues DF, Neves DR, Pinto JM, Alves MF, Fulgêncio AC. Results of patch-tests from Santa Casa de Belo Horizonte Dermatology Clinic, Belo Horizonte, Brazil, from 2003 to 2010. An Bras Dermatol. 2012;87:800-3.

6. Goldenberg A, Vassantachart J, Lin EJ, Lampel HP, Jacob SE. Nickel Allergy in Adults in the US: 1962 to 2015. Dermatitis. 2015;26:216-23.

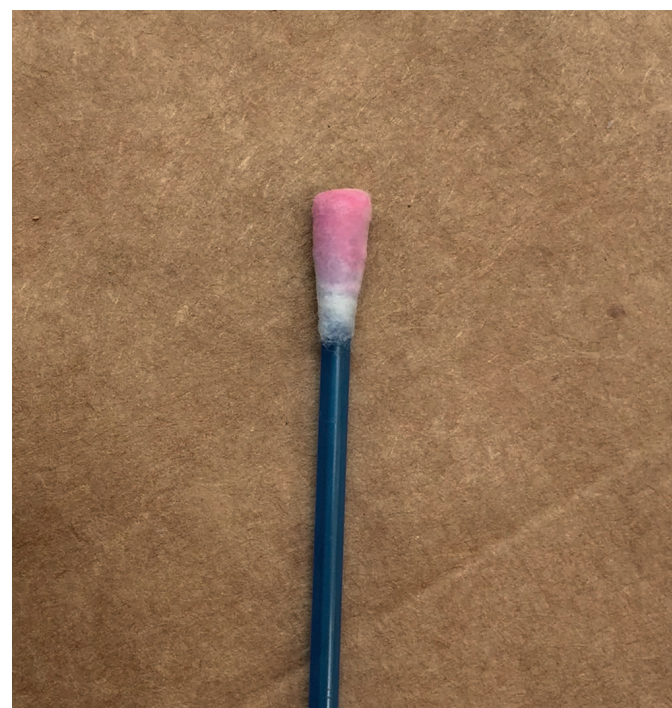

FIGURE 2: Nickel spot test with a strong positive result

Since 1994, the European Union (EU) Nickel Directive has regulated the nickel release in products. Since then, several studies have shown reduced nickel sensitization. In a population of girls of school age in Denmark, it was observed that before the legislation, $17.1 \%$ of girls with pierced ears had positive contact test for nickel. That number dropped to $3.9 \%$ after regulation. From this regulation, awareness in European children has also fallen: it is currently $9.2 \%$, compared with $28.1 \%$ in the United States $^{7}$ and $22.6 \%$ in a Brazilian study ${ }^{8}$.

We observed that the keys, objects used routinely, are responsible for exposure to these metals. In addition, through the tests used, we verified that presence of nickel and cobalt in Brazilian keys is at levels capable of inducing CAD.

Establishment of norms in Brazil to limit these metals in objects is of paramount importance for reducing the sensitization. While benefits of regulation are clear, there is little government interest in the subject. $\square$

7. Zug KA, Pham AK, Belsito DV, DeKoven JG, DeLeo VA, Fowler JF Jr, et al. Patch testing in children from 2005 to 2012: results from the North American Contac Dermatitis Group. Dermatitis. 2014;25:345-55.

8. Kobata CM. Teste de contato em crianças com eczema [tese]. São Paulo (SP) Faculdade de Medicina da Universidade de São Paulo; 2010. 45p

\section{MAILING ADDRESS: \\ Nathalie Mie Suzuki \\ R. Cesário Mota Júnior, 112 \\ Vila Buarque \\ 01221-020 São Paulo, SP \\ Brazil \\ Email:nathalie.suzuki@gmail.com}

How to cite this article: Suzuki NM, Duarte IAG, Hafner M, Lazzarini R. Nickel exposure from keys: a Brazilian issue. An Bras Dermatol. 2017;92(5):734-5. 